\title{
Advice or exercise for chronic whiplash disorders? Design of a
} randomized controlled trial

\author{
Mark J Stewart*1, Christopher G Maher ${ }^{1}$, Kathryn M Refshauge ${ }^{1}$, \\ Rob D Herbert ${ }^{1}$, Nikolai Bogduk ${ }^{2}$ and Michael Nicholas ${ }^{3}$
} \begin{abstract}
Sydney, NSW 2006 Australia
Email: Mark J Stewart* - Mark.Stewart@fhs.usyd.edu.au; Christopher G Maher - C.Maher@fhs.usyd.edu.au; Kathryn M Refshauge - K.Refshauge@fhs.usyd.edu.au; Rob D Herbert - R.Herbert@fhs.usyd.edu.au; Nikolai Bogduk - mgillam@mail.newcastle.edu.au; Michael Nicholas - miken@mail.med.usyd.edu.au

* Corresponding author
\end{abstract}

Address: ${ }^{1}$ School of Physiotherapy, Faculty of Health Sciences, University of Sydney, PO Box 170, Lidcombe, NSW, 1825, Australia, ${ }^{2}$ Newcastle Bone and Joint Institute, C/- Royal Newcastle Hospital, PO Box 664J, Newcastle, NSW 2300, Australia and ${ }^{3}$ Faculty of Medicine, University of

Published: 2I August 2003

BMC Musculoskeletal Disorders 2003, 4:18
Received: 16 May 2003

Accepted: 21 August 2003

This article is available from: http://www.biomedcentral.com/I47/-2474/4//8

(c) 2003 Stewart et al; licensee BioMed Central Ltd. This is an Open Access article: verbatim copying and redistribution of this article are permitted in all media for any purpose, provided this notice is preserved along with the article's original URL.

\begin{abstract}
Background: Whiplash-associated disorder (or "whiplash") is a common condition incurring considerable expense in social and economic terms. A lack of research on effective therapy for patients with chronic whiplash associated disorders prompted the design of the current study. The primary aim of this randomised controlled trial is to determine the effects of a physical activity program for people with chronic (symptoms of $>3$ months duration) whiplash. A secondary aim is to determine if pain severity, level of disability and fear of movement/(re)injury predict response to a physical activity program.
\end{abstract}

Methods I Design: This paper presents the rationale and design of a randomised controlled trial examining the effects of advice and individualized sub-maximal exercise programs in the treatment of whiplash associated disorders.

Discussion: This paper highlights the design, methods and operational aspects of a significant clinical trial in the area of whiplash and chronic pain.

\section{Background}

Whiplash-associated disorder (WAD or "whiplash") is a common condition incurring considerable expense in social and economic terms. In Australia, the one year incidence of whiplash is 106 per 100,000 [1]. As of June 1998, whiplash was involved in 49,344 compulsory third party claims in New South Wales (NSW) since 1995. The incurred cost of these claims was $\$ 1466.3$ M AUD [2]. Payments for loss of earnings for individuals who are tem- porarily or permanently unable to return to usual work activities are double the medical treatment costs and account for $20 \%$ of claim costs [2].

Prognosis for whiplash is generally thought to be favourable. However, a recent systematic review by Cote et al (2001) found that prognosis may vary according to the population sampled and the compensation system of the geographical area studied [3]. A large inception cohort 
study in Quebec showed that 22\% of whiplash claimants returned to their usual activities within 1 week, $53 \%$ by one month, $70 \%$ by 3 months and $97 \%$ by one year postaccident [4]. Importantly, a proportion of cases $(30 \%$ in Quebec) do not return to usual activities by 3 months. These cases account for the majority of whiplash costs [5]. Therefore, treatments that effect an early return of subjects to their usual activities, especially for those cases that fail to recover by 3 months, ie chronic whiplash, have the potential to significantly reduce social and economic costs.

In a systematic review conducted in 2000, we located 86 randomised controlled trials evaluating therapy for neck pain. Only 15 of the 86 trials identified directly looked at whiplash. Of the 15 whiplash trials, only four studied patients with chronic whiplash. Neither subcutaneous water injections [6] nor intraarticular corticosteroid injections [7] were effective for chronic whiplash. The only effective therapy is radiofrequency neurotomy [8], but the technique is invasive, is delivered in a tertiary institution, and is only indicated for subjects whose symptoms are shown by diagnostic blocks to arise from the lower cervical zygapophyseal joints. In the majority (> 75\%) of claims involving whiplash, claimants report injuries to other parts of the body [2]. Hence, for such patients, treatment of the cervical spine joints in isolation is unlikely to assist return to usual activities and therefore for many patients with chronic whiplash, neurotomy may not be a treatment option. This lack of research on effective therapy for patients with chronic whiplash associated disorders prompted the design of the current study.

There is evidence that two interventions assist patients with sub-chronic (pain of 6 weeks to 3 months duration) low back pain to return to their normal activities: 1) provision of education, reassurance and encouragement to resume normal activity [9]; and 2) prescription of a physical activity program supervised by a therapist who guides resumption of normal activities [10]. There is also evidence that physical activity programs effectively return patients with chronic low back pain to their normal activities [11]. This evidence for sub-chronic and chronic low back pain has frequently led physiotherapists to manage chronic pain from other sources with physical activity programs. Effective programs for low back pain are prescribed from a detailed assessment of the patient and include individualised, sub-maximal, progressively increased exercises to train those functions found to be inadequate for performance of work or leisure activities. Many programs incorporate principles of cognitive-behavioural therapy, eg pacing and reinforcement in the exercise sessions, and also address unhelpful beliefs such as fear of pain or re-injury. Because many of the physical, psychological and social barriers to return to normal activity for patients with chronic low back pain are also present in patients with chronic whiplash, it is possible that a physical activity program will also benefit these patients. Accordingly physical activity programs are increasingly being used by physiotherapists to manage chronic whiplash although at present there is no direct evidence to support their use.

To date only one small trial [12](30 subjects) has evaluated the use of physical activity in the management of chronic whiplash. Chiropractic treatment and 'phasic' exercise were found to reduce neck disability significantly more than chiropractic treatment and 'standard' exercise. However, neither form of exercise was clearly described and the only outcome measured was disability immediately following treatment. It is therefore unknown whether treatment effects were maintained, a critical issue for chronic conditions.

We propose to evaluate the long term effects of a physical activity program on the pain and disability associated with chronic whiplash. Best practice will be evaluated, using elements shown to be effective for chronic low back pain (eg high intensity, supervision and principles of cognitive-behavioural therapy) [11].

It is clear that the population with chronic whiplash is not homogeneous, but includes patients with varying signs, levels of pain and disability, attitudes and beliefs, who experience widely divergent social and work environments [13]. It is therefore possible that sub-populations of patients with chronic whiplash could be identified who would respond differently to physical activity programs. The prognostic factors for acute whiplash fall into three categories: socio-demographic factors, crash-related factors and clinical findings [1], but there are no data on prognostic factors for chronic whiplash, and no data on predictors of response to treatment for chronic whiplash. The proposed study will determine whether pain severity, level of disability and fear of movement/(re)injury predict treatment response in a chronic whiplash population.

This study has three primary aims:

1. The primary aim of this randomised controlled trial is to determine the effects of a physical activity program for people with chronic (symptoms of $>3$ months duration) whiplash.

2. A secondary aim is to determine if pain severity, level of disability and fear of movement/(re)injury predict response to a physical activity program. 
3. A third aim is to evaluate the psychometric properties of outcome measures that currently exist for the assessment of chronic neck pain.

\section{Methods}

This randomized controlled trial will be conducted at two treatment sites. Ethics approval has been gained from the University of Sydney Human Ethics Committee.

\section{Study population}

One hundred and eighty subjects with chronic whiplash will be recruited with the assistance of the Motor Accidents Authority (MAA) of NSW. The MAA will provide the researchers with a list of claimants who sustained a whiplash injury in a motor vehicle accident between three and six months previously. In turn, the researchers will send letters to claimants inviting them to participate in the study. Claimants who volunteer to participate and satisfy inclusion criteria will be randomly allocated to one of two treatment groups: a group that receives advice; or a group that receives a physical activity program in addition to advice. No attempt is made to blind subjects or give equivalent amounts of intervention to the two groups.

Verbal consent will be obtained over the phone in order to establish whether or not the client is appropriate for the trial. Written consent will be obtained at the initial assessment prior to obtaining any further information from the subject.

\section{Inclusion Criteria}

Volunteers will be screened by telephone and then at their first clinic appointment. To be eligible for inclusion, claimants must have presented for medical care of a whiplash associated disorder (WAD) grade I-III [3], (Table 1), within one month of the accident (such cases will be identified by excluding WAD grades 0 and IV). Claimants must also report that they are at least "mildly" disabled with respect to pre-injury status. This will be achieved by asking the question: "During the past week, how much has your neck symptoms interfered with your normal (pre-injury) activities (including work outside the home, housework, sport, recreation and family activities)?" (Response options: Not at all, Mildly, Moderately, Quite a bit, or Extremely). At trial entry subjects must have significant neck pain-related disability (indicated by a score of at least $20 \%$ on any one of the primary outcome measures, obtained at the first clinic visit, see below).

Subjects will be allowed to participate regardless of whether or not they are involved in litigation. Subject's litigation status will be recorded at the initial assessment.
Table I: Clinical Classification of Grades of WAD

\begin{tabular}{ll}
\hline GRADE & CLASSIFICATION \\
\hline $\mathbf{0}$ & $\begin{array}{l}\text { No complaint about the neck. No physical sign(s). } \\
\text { I }\end{array}$ \\
II & $\begin{array}{l}\text { Neck complaint of pain, stiffness or tenderness only. No } \\
\text { Neck complaint AND musculoskeletal sign(s). } \\
\text { Musculoskeletal signs include decreased range of motion } \\
\text { and point tenderness. }\end{array}$ \\
III & $\begin{array}{l}\text { Neck complaint AND neurological sign(s). Neurological } \\
\text { signs include decreased or absent tendon reflexes, } \\
\text { weakness and sensory deficits. } \\
\text { Neck complaint AND fracture or dislocation. }\end{array}$ \\
IV &
\end{tabular}

\section{Exclusion Criteria}

In the initial telephone interview a physical activity readiness questionnaire will be used to screen for contraindications to exercise training. Subjects will also be excluded if they have undergone neck surgery. Subjects will be screened for "red flags". "Red flags" may indicate serious pathology for which more appropriate medical intervention is required. Where red flags are identified the person will be referred for further investigation.

Volunteers not excluded by phone screening will be further screened at the first clinic visit. Volunteers will be excluded at this stage if they are classified as "severely" or "extremely severely" depressed as measured by the Depression Anxiety Stress Scale [14]. Subjects will also be excluded if they exhibit clear neurological signs. A subject will be deemed to have clear neurological signs if they have two positive tests out of three (sensation, power and reflexes) for the same spinal nerve root. All subjects will require a cervical spine $\mathrm{x}$-ray to participate. Subjects will only undergo an $\mathrm{x}$-ray investigation if films have not already been taken since the accident. Subjects will be excluded if X-rays reveal serious neck pathology such as an undiagnosed fracture or dislocation.

Subjects will be excluded from the study if they are currently undergoing treatment for their whiplash condition. Subjects will be asked to refrain from current treatment for the duration of the trial and where possible, not change current medications.

A letter will be sent to each subjects'medical practitioner advising of the subject's participation in the trial. Results of the screening process will also be given to general medical practitioners.

A record will be kept of the number of invitations to participate, the number of potential subjects who volunteer to participate, and the number of screened patients who are ineligible and the reason for their ineligibility. The 
researchers will only have access to the names and addresses of claimants and not any other information about potential subjects unless they are recruited into the study. Ineligible patients and those who choose not to participate will be referred for other care where appropriate.

A list of information on symptoms will be obtained by way of "red-flag" screening, questioning about contraindications to exercise testing and via a history taken by the treating physiotherapist. This list will be used to describe patients entering the trial and to determine whether patients with other symptoms have different outcomes.

\section{Baseline Measures}

Immediately following the screening consultation, demographic data (age, gender, level of education) and employment status will be obtained via questionnaire. Two measures of employment status will be obtained. A categorical measure of employment status (ie working full time, full duties; or working full time, some duties; or working part time, full duties; or working part time, some duties; or employed but not currently working; or employed but not currently working and undergoing retraining; or unemployed, not working) and a continuous measure of employment (ie, time since change in employment status). In addition, the following variables will be measured:

- Average pain intensity over last 24 hours on a 0-10 scale [15];

- Average bothersomeness of symptoms over last 24 hours on a $0-10$ scale [16];

- A patient-specific measure of disability (Patient-Specific Functional Scale) [17];

- A neck-specific measure of pain-related disability (Neck Disability Index) [18];

- A generic measure of health status (SF-36) [19]; and

- Fear of movement/(re)injury (Tampa Scale for Kinesiophobia) [20];

These variables will be used to describe the study sample and as baseline measures of outcomes.

Whiplash Associated Disorders incorporate a wide array of symptoms and the number of primary outcome measures noted above will be used to cover the pain, disability and quality of life changes experienced by such a population. The scales chosen are simple to score, relevant to this population and cover the aspects of WAD highlighted above. The Neck Disability Index is the most established of the scales and there is much known about the psychometric properties. The pain scales we will use are used widely and also have acceptable test properties. The SF36 is the most widely used generic health measure at present. It is easy to administer and score and has Australian Normative data for the general population.

The psychometric properties of some of the outcome measures that exist for chronic neck pain will be evaluated as there has been limited evaluation of the test properties of these measures. To investigate the responsiveness of existing functional scales for neck pain, three further questionnaires will be issued to subjects: The Functional Rating Index [21], the Copenhagen Neck Functional Disability Scale [22] and a modified Cumberland Whiplash Outcome Measure (CWOM) which is a scale developed specifically for this study based on the Proposed Core Outcomes Measure of Deyo et al [23]. These scales will be issued to each subject in order to compare the responsiveness of these scales and the Neck Disability Index to examine the internal consistency and factor structure of each measure to investigate the presence of floor and ceiling effects and to determine which scoring methods are most appropriate for each scale.

\section{Treatment allocation}

Prior to the commencement of the study, a statistician not otherwise involved in the study will generate the randomisation schedule and produce a series of sealed, consecutively numbered, opaque envelopes, each containing one subject's allocation. The statistician will retain a confidential copy of the randomisation schedule so that the accuracy of the randomisation process can be checked after the trial is completed. Randomisation will be by random permuted blocks of variable size from 6 to 10 . Allocation will occur immediately following the assessment and advice session given by the treating physiotherapist. This will ensure that the advice session is the same regardless of treatment group. At this time, the subject's name will be written across the seal of the envelope and the envelope's seal will then be broken. In this way concealment of allocation and blinding of baseline measures is ensured. Subjects will be considered to have entered the study at the time that the envelope is opened.

\section{Treatments}

After baseline measures have been collected, subjects will be seen by the treating physiotherapist who will take a complete history (including a symptom checklist), and perform a physical examination. During this assessment, current and pre-injury ability to perform work and home activities will be established and measures of upper and lower body mobility, coordination, strength and aerobic fitness will be taken. On the basis of this assessment and 
using the baseline measures, each subject will be given advice (as below). Randomisation to treatment group will occur after this advice has been given.

Subjects in the advice group will receive standardised education, reassurance and encouragement to resume light activity alone. The advice will be given in one consultation and two follow-up phone contacts. The treating physiotherapist will emphasise the favourable prognosis of whiplash and address common inaccurate beliefs about whiplash. Subjects will be told that physical activity, even that which evokes moderate pain, is unlikely to further damage the neck and back, and that excessive voluntary limitation of activity may cause muscle weakness and impede recovery. The physiotherapist will explore and discuss the patient's understanding of whiplash and attitudes and beliefs about activity resumption (eg fear of increased pain or re-injury) that may impose barriers to recovery. They will also be told that, because attempting to return to normal activities as quickly as possible may be the best possible therapy, no further treatment should be necessary. Subjects will be given a written report covering the main points of the advice session. Two and four weeks later subjects will be contacted by telephone and the standardised advice will be reinforced.

Subjects allocated to the physical activity program will receive, in addition to the standardised advice described above, an individualised physical activity program. The six-week graded program will be carried out under supervision from a physiotherapist ( 3 sessions in the first and second weeks; 2 sessions in the third and fourth weeks; and 1 session in the fifth and sixth weeks). At the first exercise session, baseline measures of upper and lower body mobility, coordination, strength and aerobic fitness will be established. From these assessments an individualised, sub-maximal, progressive activity program will be developed to train those functions found to be inadequate for performance of pre-injury work and home activities. At the same time, the physiotherapist will guide the subject's return to normal activities. Principles of cognitive-behavioural therapy (CBT) will be used by the physiotherapists in their training and supervisory roles [24]. Treating physiotherapists will receive basic training in these skills from a clinical psychologist (Author MN). The CBT principles include the encouragement of skill acquisition by modelling, setting progressive goals, self-monitoring of progress, and verbal positive reinforcement of progress. Self-reliance will be fostered by encouraging subjects to engage in problem-solving to deal with difficulties rather than seeking reassurance and advice, by encouraging relevant and realistic activity goals, and by encouraging self-reinforcement. As noted above, the physiotherapist will explore and discuss the patient's understanding of whiplash and attitudes and beliefs about activity resumption (eg fear of increased pain or re-injury) that may impose barriers to recovery. Daily physical activity at home will be encouraged and monitored using a diary to enhance compliance. Written and illustrated exercise instructions will be provided. Subjects may attend the gym at times other than their scheduled appointments to complete exercises as deemed appropriate by their therapist. Each subject will receive 12 supervised sessions. A subject is able to cancel up to 2 sessions without penalty, ie, these sessions will be made up within the 6 week period. If a subject cancels or fails to attend on more than 2 occasions, these sessions will not be made up.

Subjects in both groups will be asked not to seek other treatments and where possible not to change current medications for the six-week trial period.

Several mechanisms will be used to ensure that the trial protocol is consistently applied. Protocol manuals will be developed and staff will be trained to ensure that screening, assessment, randomisation and treatment procedures are conducted according to the protocol. Physiotherapy interventions will be provided by independent physiotherapists trained to administer the treatment protocols. An independent researcher will monitor the conduct of the study by reviewing a randomly chosen subset of assessment, randomisation and treatment procedures.

If subjects are concerned about their condition during the study, the physiotherapist will screen for potentially serious pathology and, where appropriate, refer to an adverse effects committee who may refer on to a medical practitioner. The medical practitioner will be asked not to seek information on the subject's allocation unless it is deemed necessary for medical care. After 6 weeks, interventions will cease, but both groups will be encouraged to continue their attempts to return to normal activity. Subjects will be free to seek other treatment after the experimental period, although further treatment will not be actively encouraged. Subjects will be requested to formally record the type and amount of any treatments they use.

\section{Outcome measures}

Measures of outcome will be obtained at the follow-up appointments at 6 weeks (ie, at the end of the intervention period) and at 12 months. Every attempt (within ethical constraints) will be made to obtain outcome data, regardless of subjects' compliance with trial protocols. Followup measures will be scored by a trial physiotherapist who is unaware of group allocation. However, because treatment outcomes are self-assessed by subjects, the trial cannot be assessor-blinded. 
The primary measures of clinical efficacy will be pain intensity, bothersomeness of symptoms and subject-specific disability at 6 weeks. Secondary measures of efficacy will be the same measures at 12 months, as well as 6 week and 12 month measures of SF-36 physical and mental health component summaries, the neck-specific measure of disability, and employment status. Functional Rating Index, Copenhagen Neck Functional Disability Scale and Cumberland Whiplash Outcome Measures will be collected from subjects as noted above so information on the responsiveness of these measures can be gathered. Global perceived effect will also be measured on a 10 point scale (anchors at -5 indicating vastly worse, 0 indicating unchanged, and 5 indicating completely recovered).

At both 6 weeks and 12 months, information about adverse effects of treatment will be sought from all subjects using open-ended questioning. In addition, subjects' perceptions of the credibility of intervention will be assessed at 6 weeks by questionnaire.

Compliance with the activity program will also be assessed at 6 weeks by examining attendance records and exercise diaries. Use of treatment (other than that provided as part of the trial) will also be assessed at 6 weeks and 12 months by questionnaire. Subjects will be given recommendations to formally record the amount and type of treatment that they may receive.

\section{Data analysis}

Data will be coded to enable analysis by a "blinded" statistician. The analysis will determine the size of the effect, if any, of physical activity on primary and secondary outcome variables. If physical activity is effective, further analysis will determine whether pain severity, level of disability and fear of movement/(re)injury influence the magnitude of the treatment response. Analysis will be by intention-to-treat.

Separate analyses will be conducted to determine the effect of treatment at 6 weeks and 12 months. The effects of physical activity on each of the continuous outcome measures will be analysed with multiple linear regression. Baseline values of outcome measures will be used as covariates. The coefficient of the group membership variable will provide an estimate of the effect of physical activity.

The dichotomous measure of employment status will be analysed using survival analysis methods (Cox regression, if the constant hazard assumption appears to be met, or the log rank test) for time from randomisation until return to work. This analysis will only include subjects who reported at entry to the study that they were currently not working because of whiplash-associated symptoms. The hazard ratio will provide an estimate of the effect of physical activity. Although the precision of this estimate is likely to be low because of reduced sample size, employment status is sufficiently important to warrant inclusion as a secondary outcome.

A conservative approach will be taken to analyse the predictive value of pain intensity, level of disability and fear of movement/(re)injury on treatment responses. This analysis will only proceed if at least one of the primary analyses finds statistically significant effects of physical activity (two-sided $\mathrm{p}<0.05$ ). In that case the three putative effect modifiers (pain intensity, level of disability and fear of movement/(re)injury) will be added to the regression model one at a time, along with the term for interaction with the treatment group.

The clinical and demographic data collected at baseline will be inspected and if there are any clinically and important differences in significant prognostic factors, these will be used as covariates in any analysis performed.

\section{Sample size}

Sample size was determined using the procedures described by Cohen [25]. We have assumed, probably quite conservatively, a correlation between baseline measures and outcome scores of 0.4. A sample of 90 subjects per group provides at least an $80 \%$ probability of detecting an effect of exercise of $1.5 \mathrm{~cm}$ on the $10 \mathrm{~cm}$ VAS pain intensity measure, 1.5 units on the $0-10$ symptom bothersomeness scale and $10 \%$ on the $0-100 \%$ Neck Disability Index. In our calculations, we assumed that the standard deviation (SD) of pain intensity was $2.5 \mathrm{~cm}$

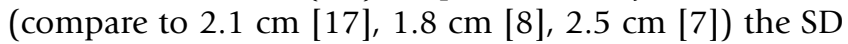
for symptom bothersomeness was 2.8 (compare to 2.8 [16]) and the SD for Neck Disability Index was 20\% (compare to $15 \%$ [26], 20\% [27], 16\% [17]), and that alpha was 0.05 . We allowed for $20 \%$ non-compliance with exercise and $10 \%$ loss to follow-up (with losses to follow-up equally distributed across compliant and non-compliant subjects).

\section{Responsiveness of Outcome Measures}

As noted above, subjects will be issued with the Functional Rating Index, the Copenhagen Neck Functional Disability Scale and the Cumberland Whiplash Outcome Measure. The responsiveness of these measures will be compared with the Neck Disability Index. There does not appear to be a consensus at present as to the best way to evaluate responsiveness [28]. In this study, two approaches will be used. Firstly we will calculate the effect size and standardised response mean. Effect size is calculated by dividing the average change between initial and follow up measures by the standard deviation of the initial measure [28]. The standardised response mean is calculated by dividing the average change between initial 
and follow up measures by the standard deviation of the change score [28]. With these indices, the larger the score the better the responsiveness. Secondly, the scores obtained by each measure will be compared with the GPE scale as an external measure of true change). Sensitivity and Specificity will be calculated for a range of cut-offs for improvement on the GPE scale and this information will be used to plot an ROC curve for the measure. The area under the ROC curve will be calculated. The area under the curve can be interpreted as the probability of correctly identifying a patient who has improved from randomly selected pairs of patients who have and have not improved. DeLong et al's [29] method of comparing ROC curves will be used.

Further analyses will be undertaken to examine the internal consistency / factor structure of each scale to investigate the presence of floor and ceiling effects of the outcome measures [30].

\section{Conclusions}

We have presented the rationale and design of an RCT examining the effects of advice and individualized submaximal exercise programmes in the treatment of whiplash associated disorders. The primary aim of this randomised controlled trial is to determine the effects of a physical activity program for people with chronic (symptoms of $>3$ months duration) whiplash. A secondary aim is to determine if pain severity, level of disability and fear of movement/(re)injury predict response to a physical activity program. The results of this trial will be presented as soon as they are available.

\section{Competing interests}

None declared.

\section{Authors' Contributions}

$\mathrm{MS}, \mathrm{CM}, \mathrm{KR}, \mathrm{RH}$ and NB were responsible for the design of the study. MN was responsible for directing the cognitive behavioural therapy aspects of the trial. MS will act as trial coordinator. All authors read and approved the final manuscript.

\section{Acknowledgements}

This project is funded by the Motor Accidents Authority of New South Wales. The authors wish to thank the staff and management of Rehab One Physiotherapy for their participation in this project.

\section{References}

I. Harder S, Veilleux $M$ and Suissa S: The effect of socio-demographic and crash-related factors on the prognosis of whiplash. Journal of Clinical Epidemiology 1998, 51:377-384.

2. Motor Accidents Authority of New South Wales: Whiplash and the NSW motor accidents scheme. Statistical Information Paper No 7. Motor Accidents Authority of New South Wales. Sydney 1999.

3. Cote P, Cassidy D, Carroll L, Frank J and Bombardier C: A systematic review of the prognosis of acute whiplash and a new con- ceptual framework to synthesize the literature. Spine 200I, 26(19):E445-E458.

4. Spitzer W, Skovron M, Salmi L, Cassidy J, Duranceau J, Suissa S and Zeiss E: Scientific monograph of the Quebec task force on whiplash-associated disorders: Redefining "whiplash" and its management. Spine 1995, 20: IS-73S.

5. Motor Accidents Authority of New South Wales: Compulsory third party claims involving whiplash. Motor Accidents Authority of New South Wales. Sydney 1994.

6. Byrn C, Borenstein P and Linder L: Treatment of neck and shoulder pain in whip-lash syndrome patients with intracutaneous sterile water injections. Acta Anaesthesiologica Scandinavica I99I, 35:52-3.

7. Barnsley L, Lord S, Wallis B and Bogduk N: Lack of effect of intraarticular corticosteroids for chronic pain in the cervical zygapophyseal joints. New England Journal of Medicine 1994, 330: 1047-50.

8. Lord S, Barnsley L, Wallis B, McDonald G and Bogduk N: Percutaneous radio-frequency neurotomy for chronic cervical zygapophyseal-joint pain. New England Journal of Medicine 1996, 335: $1721-6$

9. Indahl $A$, Velund $L$ and Reikeraas O: Good prognosis for low back pain when left untampered. A randomised clinical trial. Spine 1995, 20:473-477.

10. Lindstrom I, Ohlund C, Eek C, Wallin L, Peterson L, Fordyce $W$ and Nachemson A: The effect of graded activity on patients with subacute low back pain: a randomised prospective clinical study with an operant-conditioning behavioural approach. Physical Therapy 1992, 72:279-293.

II. Maher C, Latimer J and Refshauge K: Prescription of activity for low back pain: What works? Australian Journal of Physiotherapy 1999, 45:12I-132.

12. Fitz-Ritson $D$ : Phasic exercises for cervical rehabilitation after whiplash trauma. Journal of Manipulative and Physiological Therapeutics 1995, 18:21-24.

13. Ferrari $R$ and Russell $A$ : Epidemiology of whiplash: an international dilemma. Annals of the Rheumatic Diseases 1999, 58: I-5.

14. Lovibond $\mathrm{P}$ and Lovibond $\mathrm{S}$ : The structure of negative emotional states: comparison of the depression anxiety stress scales (DASS) with the beck depression and anxiety inventories. Behaviour Research and Therapy 1995, 33:335-343.

15. Jensen M, Turner J, Romano J and Fisher L: Comparative validity of chronic pain intensity measures. Pain 1999, 83:157-162.

16. Cherkin D, Deyo R, Street J and Barlow W: Predicting poor outcomes for back pain seen in primary care using patients' own criteria. Spine 1996, 21:2900-2907.

17. Westaway M, Stratford $P$ and Binkley J: The patient-specific functional scale: validation of its use in persons with neck dysfunction. Journal of Orthopaedic and Sports Physical Therapy 1998, 27:331-338.

18. Vernon $\mathrm{H}$ and Mior S: The Neck Disability Index: a study of reliability and validity. Journal of Manipulative and Physiological Therapeutics 1991, 14:409-415.

19. Ware J and Sherbourne C: The MOS 36-item short-form health survey (SF-36). I. conceptual framework and item selection. Medical Care 1992, 30:473-483.

20. Vlaeyen J, Kole-Snijders A, Boeren R and van Eek H: Fear of movement/(re)injury in chronic low back pain and its relation to behavioural performance. Pain 1995, 62:363-372.

21. Feise RJ and Menke M: Functional Rating Index: A new valid and reliable instrument to measure the magnitude of clinical change in spinal conditions. Spine 200I, 26:78-87.

22. Jordan A, Manniche C, Mosdal C and Hindsberg C: The Copenhagen neck functional disability scale: a study of reliability and validity. Journal of Manipulative and Physiological Therapeutics 1998 , 21:520-527.

23. Deyo R, Battie M, Beurskens A, Bombardier C, Croft P, Koes B, Malmivaara A, Roland M, Von Korff $M$ and Waddell G: Outcome measures for low back pain research: A proposal for standardised use. Spine 1998, 23(18):2003-2013.

24. Nicholas MK and Sharp TJ: Cognitive-Behavioural Programs: Theory and Application. In: Acute and Chronic Pain. International Anaesthesiology Clinics Volume 35. Edited by: Molloy A Power I. JB Lippincott, Philadelphia; 1997:155-170.

25. Cohen J: Statistical power analysis for the behavioura sciences. Hillsdale: Lawrence Erlbaum A, Publishers 21988. 
26. Riddle $D$ and Stratford $P$ : Use of generic versus region-specific functional status measures on patients with cervical spine disorders. Physical Therapy 1998, 78:95I-963.

27. Hains F, Waalen J and Mior S: Psychometric properties of the Neck Disability Index. Journal of Manipulative and Physiological Therapeutics 1998, 21:75-80.

28. Stratford P, Binkley J and Riddle D: Health status measures: Strategies and analytic methods for assessing change scores. Physical Therapy 1996, 76(10): I 109-I I 23.

29. DeLong E, DeLong D and Clarke-Pearson D: Comparing the areas under two or more correlated receiver operating characteristic curves: A nonparametric approach. Biometrics 1988, 44:837-845

30. Streiner D and Norman G: Health Measurement Scales: A practical Guide to their development and use. New York: Oxford University Press Inc 21995.

\section{Pre-publication history}

The pre-publication history for this paper can be accessed here:

http://www.biomedcentral.com/1471-2474/4/18/prepub

Publish with Bio Med Central and every scientist can read your work free of charge

"BioMed Central will be the most significant development for disseminating the results of biomedical research in our lifetime. "

Sir Paul Nurse, Cancer Research UK

Your research papers will be:

- available free of charge to the entire biomedical community

- peer reviewed and published immediately upon acceptance

- cited in PubMed and archived on PubMed Central

- yours - you keep the copyright

Submit your manuscript here:

http://www.biomedcentral.com/info/publishing_adv.asp 